\title{
Instantáneas
}

\section{Asociación entre los antecedentes familiares de diabetes y la hiperinsulinemia en niños y adolescentes aparentemente saludables}

Está reconocido que los niños y adolescentes obesos presentan factores de riesgo de enfermedad coronaria y de trastornos metabólicos de la glucosa. Los niveles de insulina tienen una correlación directa con la obesidad, en particular con la obesidad abdominal, por lo que los índices de secreción de insulina, como la hiperinsulinemia, pueden ayudar a evaluar el riesgo de diabetes y de enfermedad cardiovascular en los niños.

Algunos estudios realizados en adultos han demostrado que los antecedentes familiares de diabetes tipo 2 (AF-D) están asociados con la hiperinsulinemia, independientemente de la obesidad. Es conocido que no todas las personas obesas muestran resistencia a la insulina y que muchas personas no obesas pueden presentar una disminución de la sensibilidad a la insulina. Estos hallazgos indican que otros factores, como los AF-D, pueden desempeñar un papel importante en el desarrollo de la resistencia a la insulina, además de la obesidad.

En este estudio se evaluó la posible asociación entre los AF-D y la hiperinsulinemia en ayunas en niños y adolescentes sanos. Para ello se realizó un estudio transversal basado en la población con muestreo por conglomerados en dos etapas. En la primera etapa se seleccionó una muestra aleatoria de escuelas primarias y de enseñanza media del estado de Durango, México, y en la segunda se seleccionaron al azar niños de 10 a 14 años de esas escuelas. Un pediatra determinó el buen estado de salud de los niños (índice de Tanner 1 ó 2). Los antecedentes familiares de hipertensión arterial y los AF-D se establecieron mediante el examen médico detallado y pruebas de laboratorio (medición de la tensión arterial, glucemia en ayunas y prueba oral de tolerancia a la glucosa) a ambos padres y mediante el análisis de sus historias clínicas. Los padres que llevaban tratamiento antihipertensivo se consideraron hipertensos. Los antecedentes familiares de obesidad se determinaron mediante la medición directa del peso, la circunferencia de la cintura, la masa de grasa corporal y el porcentaje de grasa corporal de ambos padres. La obesidad se definió como el índice de masa corporal de $30 \mathrm{~kg} / \mathrm{m}^{2}$ o mayor.
Este es el primer estudio en el que los antecedentes familiares se obtienen mediante la medición directa de ambos padres y el análisis de sus historias clínicas. Los resultados demostraron que los AF-D están asociados de forma independiente con la hiperinsulinemia, incluso en niños y adolescentes con peso corporal normal hijos de padres diabéticos. Además, los niños y adolescentes con hiperinsulinemia, independientemente de su condición con respecto a la obesidad, presentaron un perfil lipídico desfavorable y se ubicaron en el percentil superior de tensión arterial, lo que puede indicar que los jóvenes con hiperinsulinemia también pueden presentar factores de riesgo de enfermedad cardiovascular.

Este estudio también demostró que la resistencia a la insulina en los niños y adolescentes con AF-D es significativamente mayor que la de sus coetáneos que no tienen AF-D. Tanto la hiperinsulinemia como la resistencia a la insulina contribuyen a desarrollar la diabetes tipo 2, sin embargo, no está claro aún si la hiperinsulinemia en los hijos de padres con diabetes tipo 2 es una compensación temprana de la disminución de la sensibilidad a la insulina o es el trastorno primario.

Debido a que la obesidad en la niñez es cada vez más frecuente y está fuertemente asociada con la resistencia a la insulina, las políticas de salud se centran en el tamizaje de los niños y adolescentes obesos. Sin embargo, estos resultados demuestran que la hiperinsulinemia y el perfil lipídico desfavorable relacionado con ella también están fuertemente asociados con los AF-D y se encuentran con frecuencia en niños con peso corporal normal. Estos resultados podrían apoyar la conveniencia de ampliar el tamizaje de factores de riesgos cardiovasculares y metabólicos a niños y adolescentes que no presentan obesidad pero tienen AF-D. (RodríguezMorán M, Guerrero-Romero F. Hyperinsulinemia in healthy children and adolescents with a positive family history for type 2 diabetes. Pediatrics. 2006; 118(5):E1516-22.) 